\title{
S:Pububsaúde
}

ARTIGO ORIGINAL

\section{Determinação da frequência de micronúcleos em células esfoliativas da mucosa oral em indivíduos fumantes e etilistas}

\section{Determination of the frequency of micronuclei in exfoliative cells of the oral mucosa in smokers and alcoholics}

Luan Aércio Melo Maciel ${ }^{1} \bullet$, Deusiane Ribeiro da Silva ${ }^{10}$, Suany Vasconcelos da Silva $^{1} \bullet$, Talita Castro ${ }^{1} \bullet$, Luana Santos da Costa $^{1}{ }^{\bullet}$, Thais Sena Trolly ${ }^{1} \bullet$, Isabela Natilde Costa Goes ${ }^{1} \bullet$, Samaroni Brelaz Feitosa ${ }^{2}$, Albino Luciano Portela de Sousa $^{10}$

${ }^{1}$ Instituto Esperança de Ensino Superior (IESPES), Santarém-PA, Brasil. ²Universidade do Estado do Pará (UEPA), Santarém-PA, Brasil.

\section{E-mail: luanaercio@hotmail.com}

Como citar: Maciel, L.A.M., Silva, D.R. Silva, S.V., Castro, T., Costa, L.S., Trolly, T.S., Goes, I.N.C., Feitosa, S.B., Sousa, A.L.P. 2019. Determinação da frequência de micronúcleos em células esfoliativas da mucosa oral em indivíduos fumantes e etilistas, 2, aoo6. DOI: https://dx.doi.org/10.31533/pubsaude2.a 006

Recebido: 24 mai. 2019.

Revisado e aceito: 24 jun. 2019

Conflito de interesse: OS autores declaram, em relação aos produtos e companhias descritos nesse artigo, não ter interesses associativos, comerciais, de propriedade ou financeiros que representem conflito de interesse.

Licenciamento: Este artigo é publicado na modalidade Acesso Aberto sob a licença Creative Commons Atribuição 4.0 (CC-BY 4.0).
R e s u mo . O fumo tem sido descrito como o principal fator de risco no desenvolvimento de lesões malignas e pré-malignas na mucosa bucal. O consumo de álcool, por outro lado, é citado como forte agente potencializador no desencadeamento de lesões cancerosas. O estudo objetivou avaliar a frequência de micronúcleos em células esfoliativas da mucosa oral de indivíduos fumantes e etilistas, comparando-os com indivíduos não expostos. Quarenta voluntários foram selecionados e distribuídos em quatro grupos contendo indivíduos expostos ao fumo e ao álcool e não expostos. Para determinação da frequência de micronúcleos empregou-se a técnica do ensaio de micronúcleos em células da mucosa oral. As células examinadas foram coletadas com auxílio de uma escovinha citobrush e coradas com as técnicas de Feulgen/Fast-green. $\mathrm{Na}$ presente pesquisa a frequência de micronúcleos nos grupos expostos a genotoxicidade do fumo e álcool foi maior $(\mathrm{P}<0,01)$ quando comparados ao grupo controle. A associação entre álcool e fumo potencializou o aparecimento de micronúcleo nos indivíduos usuários contínuos dessas duas substâncias $(\mathrm{P}<0,05)$. Fumantes e consumidores de bebidas alcoólicas apresentam maiores quantidades de danos citotóxicos em células da mucosa oral ocasionados pela ação das substâncias cancerígenas presentes na fumaça do cigarro e no álcool.

P a l a v r a s - ch a ve : micronúcleo, genotoxicidade, câncer, fumo, álcool. 
A b s t r a c t . Smoking has been described as the main risk factor in the development of malignant and premalignant lesions in the oral mucosa, and alcohol consumption, on the other hand, is cited as a strong potential agent in triggering cancerous lesions. The study aimed to evaluate the frequency of micronuclei in exfoliative cells of the oral mucosa of smokers and alcoholics, comparing them with non-exposed individuals. Forty volunteers were selected and distributed in 4 groups containing individuals exposed to smoking and alcohol and not exposed. To determine the micronucleus frequency, the micronucleus assay technique was used in oral mucosal cells. The cells examined were collected using cytobrush brush and stained with Feulgen/Fast-green techniques. In the present study, the frequency of micronuclei in the groups exposed to tobacco and alcohol genotoxicity was significantly higher $(\mathrm{P}<$ 0.01) when compared to the control group. The association between alcohol and tobacco increased the appearance of micronucleus in the continuous users of these two substances $(\mathrm{P}<$ 0.05). Smokers and consumers of alcoholic beverages present higher amounts of cytotoxic damage to oral mucosa cells caused by the action of carcinogens present in cigarette smoke and alcohol.

K e y w o r d s : micronucleus, genotoxicity, cancer, smoking, alcohol.

\section{INTRODUÇÃO}

A população está continuamente exposta a diversas substâncias tóxicas de forma consciente ou inconsciente. Entre estas substâncias, o álcool e o fumo figuram entre os mais comuns (Barbon et al., 2015). O fumo tem sido descrito como o principal fator de risco no desenvolvimento de lesões malignas e pré-malignas na mucosa bucal (Carrard et al., 2007). O consumo de álcool, por outro lado, é citado como forte agente potencializador no desencadeamento de lesões cancerosas, promovendo alterações efetivas em células da mucosa bucal (Reis et al., 2002).

O câncer oral é considerado pela Organização Mundial da Saúde (OMS) a forma mais comum de neoplasia de cabeça e pescoço, com incidência de aproximadamente 390.000 novos casos a cada ano, representando o sexto tipo de câncer mais comum em todo o mundo (Santos et al., 2010).

No Brasil, os cânceres de lábio e cavidade oral são o quinto tipo mais incidente entre homens e o $12^{\circ}$ mais incidente entre mulheres (excluído o câncer de pele não melanoma), sendo estimados cerca de 15 mil novos casos desta doença por ano (Brasil, 2016). São muitos os fatores relacionados com a etiologia do câncer oral; porém, o etilismo e o 
tabagismo são descritos como os maiores responsáveis pelo desenvolvimento do câncer de boca (Santos et al., 2010).

O dano provocado pelo consumo de álcool, e seu efeito sinérgico com o fumo, na mucosa oral pode ser resultado de sua ação direta, pela sua presença na corrente sanguínea ou de sua atuação sobre outros sistemas (Carrard et al., 2007). O monitoramento de populações expostas ao fumo e álcool deve ser feito empregando-se testes citogenéticos para avaliar os danos cromossômicos causados pelos efeitos genotóxicos destas substâncias (Batista \& Campos Júnior, 2015).

Anteriormente ao processo de carcinogênese, as células epiteliais exibem durante o ciclo celular mitótico o aparecimento de micronúcleos, que são fragmentos de cromatina procedentes de mitoses aberrantes que permanecem próximas ao núcleo celular e sua frequência tem sido utilizada para avaliar o grau de injúria genotóxica ao qual células animais estão expostas (Giovanini et al., 2009). O teste do micronúcleo (MN) é uma ferramenta de auxílio ao processo de indicação de lesão préneoplásica pela mensuração da quantidade dessas alterações encontradas nas células com maior exposição a agentes mutagênicos (Faria \& Braga, 2015). Este teste tem sido empregado para o monitoramento de danos genotóxicos e citotóxicos, o qual consiste em um ensaio usado para rastrear a genotoxicidade, avaliando a evidência de micronúcleos e outras anormalidades nucleares no citoplasma de células em interfase (Salem et al., 2018).

O presente estudo objetivou avaliar a frequência de micronúcleos em células esfoliativas da mucosa oral de indivíduos fumantes e etilistas, comparando-os com indivíduos não expostos.

\section{MATERIAL E MÉTODOS}

\section{TIPO DE ESTUDO}

Trata-se de uma pesquisa explicativa e experimental de abordagem quantitativa que visou identificar a frequência de micronúcleos em indivíduos fumantes e etilistas através do teste de micronúcleo. A pesquisa explicativa visa identificar os fatores que determinam ou que contribuem para a ocorrência dos fenômenos. O método experimental consiste em investigações de pesquisa empírica cujo objeto principal é o teste de hipóteses que dizem respeito a relações de tipo causa-efeito. Os métodos quantitativos podem demonstrar que as variáveis são relacionadas de forma sistemática, mas não fornecem uma compreensão clara do por que estão relacionadas (Gil, 2008; Lakatos \& Marconi, 2010; Polit \& Beck, 2011). 
Utilizou-se o teste do micronúcleo, pois este se destaca entre os ensaios biológicos devido sua especificidade e sensibilidade para o monitoramento de indivíduos sob risco carcinogênico. O teste aponta que em células da mucosa oral uma frequência elevada de micronúcleos é indicativa de altas taxas de mutação e tem sido relacionada ao desenvolvimento de carcinomas neste tecido (Rodrigues et al., 2013).

\section{PARTICIPANTES DA PESQUISA}

Fizeram parte do estudo 40 voluntários provenientes do município de Santarém-PA, de ambos os gêneros que foram divididos em quatro grupos contendo 10 indivíduos cada. Foram inclusos no grupo 1 indivíduos sem nenhum tipo de exposição, ou seja, grupo controle; grupo 2 composto por apenas fumantes; grupo 3 constituído por somente etilistas e grupo 4 formado por voluntários fumantes e etilistas. Os voluntários foram separados em diferentes grupos com o intuito de verificar se a exposição prévia as substâncias cancerígenas presentes no fumo e no álcool são determinantes no aumento da quantidade de micronúcleos entre fumantes e etilistas comparados aos indivíduos sem esses hábitos. A quantidade de micronúcleos encontrados nos grupos expostos foi comparada ao grupo controle.

A separação dos voluntários em cada categoria ocorreu por meio do preenchimento de uma entrevista estruturada na qual foi aplicada pelos próprios autores, onde se coletou informações acerca da idade, gênero, exposição prévia a agentes mutagênicos como fumo, álcool, raio X, uso de aparelho ortodôntico, antisséptico bucal e medicamentos. Foram incluídos neste estudo indivíduos que se enquadraram em apenas uma das variáveis estudadas, indivíduos etilistas com consumo médio de 14 doses semanais de bebidas alcóolicas, fumantes com hábito de fumar pelo menos um cigarro por dia.

$\mathrm{Na}$ constituição do grupo controle selecionaram-se indivíduos considerando-se a situação ocupacional. Fizeram parte deste grupo alunos de uma universidade, estes não apresentavam contato com possíveis agentes de ação genotóxica estabelecida, reduzindo-se assim fatores de conflito.

Todos os voluntários assinaram o Termo de Consentimento Livre e Esclarecido conforme preconiza a resolução 466/2012 do Ministério da Saúde. A pesquisa foi aprovada pelo Comitê de Ética em Pesquisa (CEP) do Instituto Esperança de Ensino Superior (IESPES) sob o Parecer $n^{\circ}$ 3.197.067.

AVALIAÇÃo DE MICRONÚCLEOS: COLETA DO MATERIAL, PREPARO DA LÂMINA E ANÁLISE 
O teste do micronúcleo em células da mucosa bucal foi executado conforme a metodologia usada por Souto et al. (2010).

As coletas foram realizadas após os voluntários da pesquisa responder uma entrevista estruturada contendo 10 perguntas. Posteriormente foi realizada a coleta das células epiteliais da mucosa oral dos participantes, com auxílio de uma escova citológica do tipo citobrush, onde as células raspadas foram espalhadas sobre lâminas previamente limpas com álcool $99,5^{\circ} \mathrm{GL}$. Os esfregaços foram realizados à temperatura ambiente sendo posteriormente fixado com fixador citológico spray. Não houve bloqueio da citocinese nas células investigadas no presente estudo.

As lâminas sofreram hidrólise ácida do DNA com solução de ácido clorídrico $(\mathrm{HCl}) 10 \%$ por 2 minutos a temperatura ambiente, coradas com solução de fucsina básica por 10 minutos ao abrigo da luz. Logo depois foram enxaguadas levemente com água para retirar o excesso de corante. Posteriormente as lâminas foram contra coradas com a solução de Fast Green por 1 minuto, passado este tempo, as lâminas foram enxaguadas com água para retirada de excesso de corante.

Para a quantificação de micronúcleos analisou-se 2000 células por indivíduo, em teste cego. Deste modo, objetivou-se manter a imparcialidade do pesquisador sobre os resultados. Utilizou-se na análise microscópica objetiva de imersão com lente de aumento 100x para análise em microscópio óptico. Os parâmetros adotados para contagem de micronúcleos foram:

-Tamanho menor do que $1 / 3$ do núcleo;

- Padrão da estrutura e coloração da cromatina idêntica ao do núcleo;

- Nenhuma ligação como o núcleo (Figura 1);

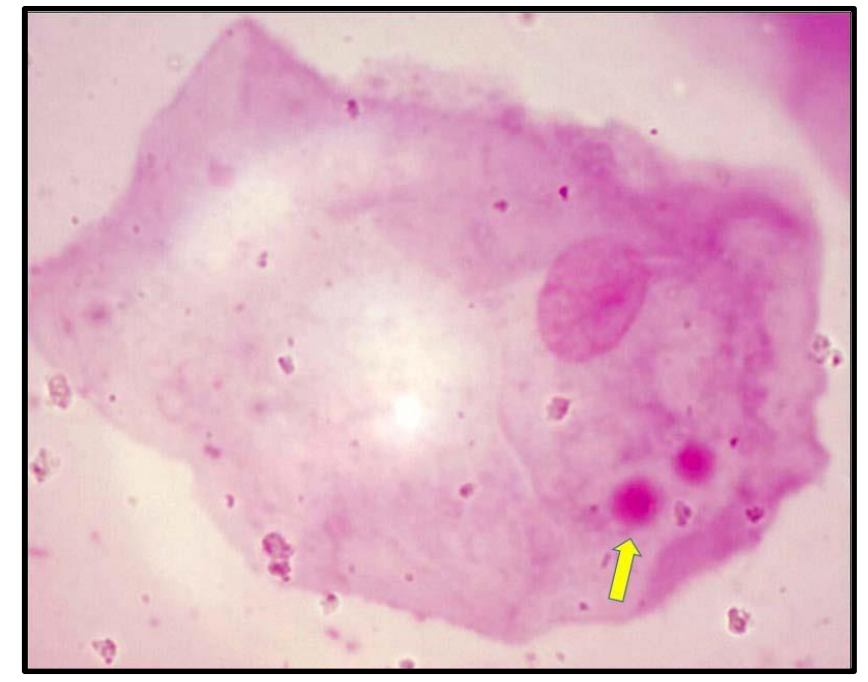

Figura 1. Fotomicrografia de uma célula esfoliativa da mucosa oral do presente estudo contendo micronúcleo. 
Os dados da análise da frequência de micronúcleos foram apresentados mostrando-se os valores da diferença encontrada entre a média e desvio padrão da média (DP). A comparação entre grupos foi realizada através do teste ANOVA e Teste de Tukey, os quais utilizam a diferença entre as médias obtidas na frequência de micronúcleos entre os grupos investigados, sendo que as diferenças foram consideradas significativas quando $\mathrm{P}<0,05$. Os dados foram analisados utilizando o software Bioestat versão 5.3.

\section{RESULTADOS E DISCUSSÃO}

Fizeram parte do estudo 40 indivíduos de ambos os gêneros com idade entre 18 e 86 anos. Os achados das alterações nucleares demonstraram que em todos os grupos analisados houve a presença de micronúcleos. Maior frequência de micronúcleos foi encontrada no grupo composto por indivíduos fumantes e etilistas (125 micronúcleos), seguido do grupo de fumantes (86 micronúcleos), em terceiro o grupo formado por etilistas (74 micronúcleos) e o grupo controle, com voluntários não expostos apresentou menor quantidade de micronúcleos (12) (Tabela 1).

Estes resultados apontam que indivíduos com hábito de consumir cigarros e ingerir bebidas alcoólicas de forma frequente possuem maiores taxas de danos genotóxicos em virtude da exposição aos contaminantes presentes na composição do fumo e do álcool.

Tabela 1. Frequência de micronúcleos em células da mucosa bucal dos participantes.

\begin{tabular}{lccc}
\hline \multicolumn{1}{c}{ Grupos } & $\begin{array}{c}\text { Número de } \\
\text { indivíduos }\end{array}$ & Micronúcleos & Média e desvio padrão \\
\hline Controle & 10 & 12 & $1,2 \pm 0,8$ \\
Fumantes & 10 & 86 & $8,6 \pm 3,7$ \\
Etilistas & 10 & 74 & $7,4 \pm 2,1$ \\
Fumantes e etilistas & 10 & 125 & $12,5 \pm 3,7$ \\
\hline
\end{tabular}

Andrade et al. (2015) analisaram os fatores associados ao câncer de boca em uma população do Nordeste Brasileiro e destacam o tabagismo e etilismo como os principais causadores do câncer de boca. Além disso, apontam que o consumo sinérgico de tabaco e álcool aumenta o risco de desenvolver essa neoplasia. Barbon et al. (2015) investigaram a presença de micronúcleos em consumidores de álcool e cigarro e destacam que no grupo exposto, a maioria dos indivíduos apresentou entre um e dez micronúcleos a cada 200 células analisadas. Estes resultados indicam que o álcool e o cigarro podem representar condições suficientes para 
desenvolver micronúcleos, como observado entre os sujeitos que se declararam usuários destas substâncias. Em um estudo realizado no município de Alta Floresta- MT detectou que a quantidade de micronúcleos em uma população de fumantes e fumantes passivos é maior do que no grupo de não fumantes, e que o teste do micronúcleo se traduz num importante marcador intermediário para avaliar o grau de exposição da mucosa bucal a carcinógenos (Tiago \& Conde, 2011).

A comparação da frequência de micronúcleos entre os grupos mostrou aumento significativo entre os voluntários dos grupos formados por fumantes e etilistas, em que a frequência de micronúcleos nos grupos expostos foi significativamente maior ( $\mathrm{P}<0,01$ - Anova e Tukey) quando comparados ao grupo controle não exposto. A associação entre álcool e fumo potencializou $(\mathrm{P}<0,05)$ o aparecimento de micronúcleo nos indivíduos usuários contínuos dessas duas substâncias (Figura 2).

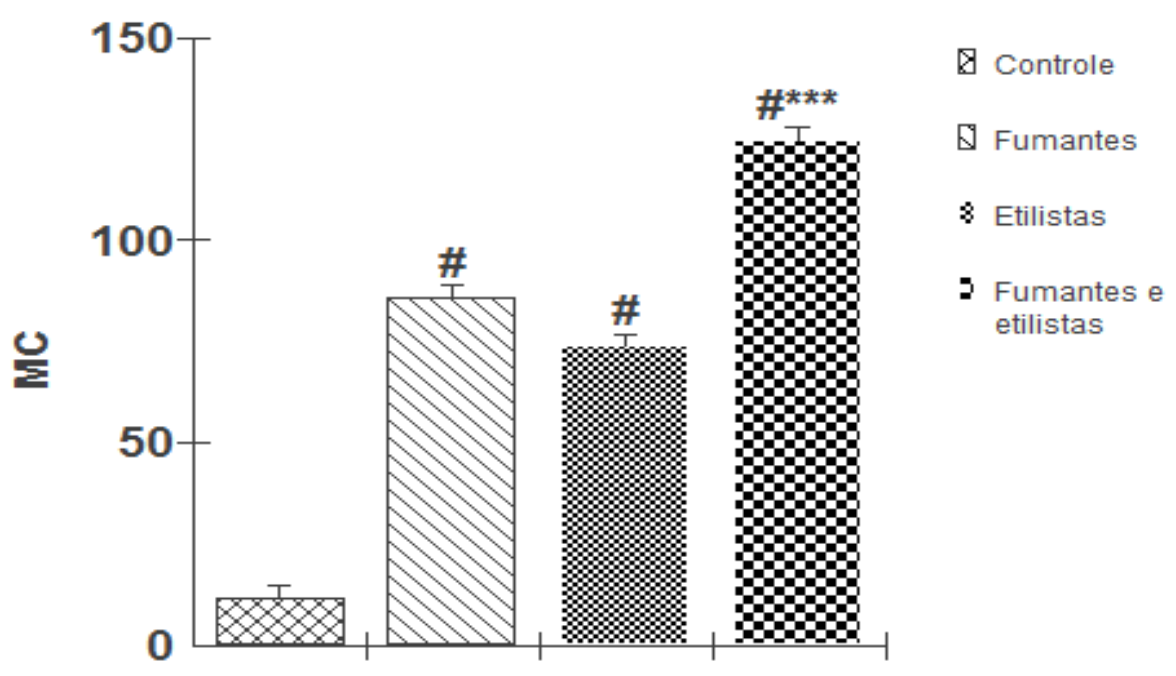

Figura 2. Análise estatística da distribuição da frequência de micronúcleos entre os grupos investigados pela da variação da média e desvio padrão utilizando-se o teste ANOVA e TUKEY, empregando-se índice de significância $(\mathrm{P}<0,05)$. \#Grupos de indivíduos fumantes, etilistas e fumantes etilistas comparados ao grupo controle exibindo aumento na frequência de micronúcleos com valor de $\mathrm{P}<0,01$. **** Grupos de indivíduos fumantes etilistas, comparado aos grupos de indivíduos somente fumantes e somente etilistas evidenciando aumento significativo da frequência de micronúcleos com valor de $\mathrm{P}<0,05$.

Resultado semelhante ao encontrado no estudo realizado por Batista \& Campos Júnior (2015) no qual o número de micronúcleos encontrados foi mais frequente no grupo exposto $(\mathrm{P}<0,05)$ comparado ao grupo controle, tanto para a frequência de micronúcleos quanto para as células micronucleadas.

$\mathrm{Na}$ fumaça do tabaco já foram detectadas mais de 60 substâncias carcinogênicas, incluindo Hidrocarbonetos policíclicos aromáticos que atuam como iniciadores promotores da carcinogênese devido à exposição contínua ao calor desprendido pela combustão do fumo (Campos et al., 
2017). Martins \& Boschini Filho (2003) utilizaram o teste do micronúcleo em células esfoliativas da mucosa bucal demonstrando que existe associação significativa entre o hábito de fumar e a presença de micronúcleos e outros tipos de alterações celulares neste tipo de células. Andrade et al. (2005) apontam que o fumo e o uso excessivo de álcool, comuns a inúmeros processos patológicos, têm tido especial interesse na etiopatogenia do câncer de boca e do trato aero digestivo. O mecanismo que associa esses agentes a um aumento do risco fundamenta-se em suas composições químicas e na interação com outros fatores.

O teste do micronúcleo em estudos epidemiológicos pode aumentar o conhecimento a respeito do potencial genotóxico de agentes ambientais e elucidar mecanismos da ação carcinogênica (Martins \& Boschini Filho, 2003).

\section{CONCLUSÃO}

Fumantes e consumidores de bebidas alcoólicas apresentam maiores quantidades de danos citotóxicos em células da mucosa oral ocasionados pela ação das substâncias cancerígenas presentes na fumaça do cigarro e no álcool. No presente estudo, estes danos foram evidenciados por maiores quantidades de micronúcleos em indivíduos com hábito de fumar e consumir bebidas alcoólicas constantemente quando comparados aos indivíduos sem exposição prévia aos contaminantes investigados, sendo que tal exposição aumenta o risco de carcinogênese no público exposto.

\section{REFERÊNCIAS}

Andrade, J. O. M., Santos, C. A. d. S. T., \& Oliveira, M. C. 2015. Fatores associados ao câncer de boca: um estudo de caso-controle em uma população do Nordeste do Brasil. Revista Brasileira de Epidemiologia, 18, 894-905.

Andrade, M. G. S., Reis, S. R. A., Robinson, W. M., \& Borges-Osório, M. R. 2005. Micronúcleo: um importante marcador biológico intermediário na prevenção do câncer bucal. Revista Odonto Ciência, 20(48), 137-141.

Barbon, F. J., Wiethölter, P., Burille, A., Casarin, D., Solda, C., Flores, R. A., \& Sabadin, C. E. S. 2015. Micronúcleos em fumantes e etilistas. Journal of Oral Investigations, 3(2), 42-45.

Batista, C. R., \& Campos Júnior, E. O. 2015. Avaliação da genotoxicidade em células de pacientes fumantes e não fumantes por meio do teste do micronúcleo. Revista $\mathrm{GeTeC}, 3(6), 49-58$.

Brasil, Ministério da Saúde. Instituto Nacional de Câncer José Alencar Gomes da Silva. 2016. Informativo Deteç̧ão Precoce. Boletim, 2016; ano 7, n1, 1-8.

Campos, A. A. B., Gonçalves, P. C., \& Noventa, M. A. 2017. Efeitos genotóxicos do uso crônico do fumo na mucosa oral. Investigação, 16(8), 82-86. 
Carrard, V. C., Ferreira, L. A., Lauxen, I. d. S., Costa, C. H., \& Rados, P. V. 2007. Teste dos Micronúcleos: Um biomarcador de dano genotóxico em células descamadas da mucosa bucal. Revista da Faculdade de Odontologia de Porto Alegre, 48(1/3), 77-81.

Faria, L. E. M., \& Braga, J. R. M. 2015. Aplicação do teste de micronúcleo para avaliação de potencial genotóxico em epitélio oral de estudantes universitários. Revista Atualiza Saúde, 1(11), 35-41.

Gil, A. C. 2008. Como elaborar projetos de pesquisa. São Paulo, SP: Atlas.

Giovanini, A. F., Vieira, D. C., Franco, L. B., Cesar, J., Zielak, E. P., \& Gonzaga, C. C. 2009. Análise de micronúcleo em citologia esfoliativa de lesões leucoplásicas em boca. Perspective Oral Science, 1, 19-23.

Lakatos, E. M., \& Marconi, M. A. 2010. Fundamentos da metodologia científica Fundamentos da metodologia científica (Vol. 1, pp. 312 p.). São Paulo, SP: Altas.

Martins, K. F., \& Boschini Filho, J. 2003. Determinação da frequência de micronúcleos e outras alterações nucleares em células da mucosa bucal de indivíduos não-fumantes e fumantes. Revista da Faculdade de Ciências Médicas de Sorocaba, 5(1), 43-53.

Polit, D. F., \& Beck, C. T. 2011. Fundamentos de pesquisa em enfermagem: avaliação de evidências para a prática da enfermagem. Porto Alegre, RS: Artmed Editora.

Reis, S. R. d. A., Sadigursky, M., Andrade, M. G. S., Soares, L. P., Santo, A. R. d. E., \& Vilas Bôas, D. S. 2002. Efeito genotóxico do etanol em células da mucosa bucal. Pesquisa Odontológica Brasileira, 16(3), 221-225.

Rodrigues, R. O., Moreira, L. M. A., Silva, E. R. J., Trindade, J. P. B., Rocha, L. M., Santo, L. D. d. E., \& Moreira, S. C. 2013. Ensaio do micronúcleo como indicador de genotoxicidade em indivíduos com albinismo e histórico prévio de câncer de pele. Revista de Ciências Médicas e Biológicas, 2.

Salem, E., El-Garawani, I., Allam, H., EL-AAL, B. A., \& Hegazy, M. 2018. Genotoxic effects of occupational exposure to benzene in gasoline station workers. Industrial health, 56(2), 132-140.

Santos, G. L., Freitas, V. S., Andrade, M. d. C., \& Oliveira, M. C. 2010. Fumo e álcool como fatores de risco para o câncer bucal. Odontologia Clínico-Científica, 9(2), 131-133.

Souto, R., Borges, F. R., Cruz, D. M., Vilanova-Costa, C. A. S. T., \& Cruz, A. D. 2010. O teste de micronúcleo como ferramenta qualitativa de dano genético: aspectos citotécnicos. Revista de Ciências Ambientais e Saúde, 37(4), 297-307.

Tiago, A. V., \& Conde, R. F. (2011). Teste de micronúcleo na mucosa oral de fumantes, não fumantes e fumantes passivo em alta floresta mato grosso. Paper presented at the Seminário de Extensão Universitária, Alta Floresta, Mato Grosso.

\section{MINICURRÍCULO}

LUAN AÉRCIO MELO MACIEL Enfermeiro, Instituto Esperança de Ensino Superior (IESPES), Santarém-PA, Brasil.

DEUSIANE RIBEIRO DA SILVA Discentes do curso de graduação em Biomedicina, Instituto Esperança de Ensino Superior (IESPES), Santarém-PA Brasil. 
SUANY VASCONCELOS DA SILVA Discentes do curso de graduação em Biomedicina, Instituto Esperança de Ensino Superior (IESPES), Santarém-PA Brasil.

TALITA CASTRO Discentes do curso de graduação em Biomedicina, Instituto Esperança de Ensino Superior (IESPES), Santarém-PA Brasil.

LUANA SANTOS DA COSTA Enfermeira, Instituto Esperança de Ensino Superior (IESPES), Santarém-PA, Brasil.

THAIS SENA TROLLY Enfermeira, Instituto Esperança de Ensino Superior (IESPES), Santarém-PA, Brasil.

ISABELA NATILDE COSTA GOES Enfermeira, Instituto Esperança de Ensino Superior (IESPES), Santarém-PA, Brasil.

SAMARONI BRELAZ FEITOSA Docente, Universidade do Estado do Pará (UEPA), Santarém-PA, Brasil.

ALBINO LUCIANO PORTELA DE SOUSA Professor associado, Instituto Esperança de Ensino Superior, Santarém-PA, Brasil. 